\title{
An optimal control model of crop thinning in viticulture
}

\author{
Guenter H. Schamel and Stefan F. Schubert \\ Free University of Bozen-Bolzano, Faculty of Economics and Management, 39100 Bozen-Bolzano, Italy
}

\begin{abstract}
We develop an economic model of cluster thinning in viticulture to control for grape quantity harvested and grape quality, applying a simple optimal control model with the aim to raise grape quality and related economic profits. The model maximizes vineyard owner profits and allows to discuss two relevant scenarios using a phase diagram analysis: (1) when the initial grape quantity is sufficiently small, thinning grapes will not be optimal and (2) when the initial grape quantity is high enough, it is optimal to thin grapes from the beginning of the relevant planning horizon and to reduce the quantity over time until the stock of grapes arrives at its optimum. Depending on the model's parameters, the "stopping time" for thinning grapes is reached sooner or later. After the stopping time, grape quantity evolves solely according to natural decay. The results relate to observed dynamics in viticulture and for other horticultural crops.
\end{abstract}

\section{Introduction}

Crop thinning (i.e. yield management) is a standard instrument to maximize economic returns derived from arable land use. In agriculture, horticulture, and forestry, we find different ways and methods to do crop thinning. In horticulture, for example, excessive fruit (e.g. apples, peaches) is removed from trees in order to raise the overall quality of the remaining fruit and thus economic returns from an orchard [1]. Thinning and pruning is also used in silviculture in order to optimize the growth rates and wood quality of the remaining trees [2]. In agriculture, sugar beets are thinned in order to provide enough space for the remaining beets to flourish and for wheat, the literature reports positive thinning effects on kernel weight up to $20 \%$ [3].

In viticulture, crop thinning is typically referred to as cluster thinning and it is well known that higher grape yields may have a negative impact on grape and wine quality $[4,5]$. Because vines have limited productive capacities, excess grapes are removed from the vine in order to raise the quality of the grapes harvested. The vast majority of high quality / high priced wines around the world are produced from low yielding vineyards. Site specific characteristics such as soil fertility and weather conditions will of course also have a significant impact on yields. For example, steep vineyard sites (e.g. on the Mosel river in Germany) are known to have very little organic material. Such vineyard sites often have very low yields but their grapes develop extreme flavours, tannins, and levels of sugar and acidity which will turn into high quality crafted wines. More fertile soils can also produce excellent wines, but vineyard managers often have to engage in a number of corrective measures including cluster thinning to make it happen. Besides pruning new shoots and excess leaves to guarantee proper exposure to sunlight, it is often necessary to also cut excess grapes during the growing season in an effort to correct otherwise high yields and to harvest higher quality grapes later in the season.
From an economic point of view, cluster thinning (or crop thinning in general) can be regarded as tradingoff quantity for wine quality with substantial economic implications. While high yields may produce quantities at lower wine qualities which can also be economical within certain price ranges, producers may need to meet specific quality standards at least partially through lowering yields due to regional wine quality regulations (e.g. AOC or DOC). Producers must find the correct balance between quantity, quality and the ensuing prices obtainable in the market. This paper aims to develop a simplified theoretical model to analyse this relationship optimizing the economic outcome using a dynamic optimal control model.

\section{Literature review}

As a starting point for our modelling approach, a large number of economic applications related to optimal thinning can be found in the forest literature. Schreuder [6] is an early application of optimal control theory (calculus of variations) to problems of optimal thinning and rotation length for forests. Medhurst [7] found that "more frequent, less severe pruning of Acacia melanoxylon (a highly adaptive tree species) may be preferable in terms of maintaining ...vigour", and that "intensive management is required to maximise Acacia melanoxylon growth and optimise stem form." Sourcy [8] points out that thinning of forest stands is often assumed to improve overall stand profitability. Lu and Gong [9] develop a model of optimal stocking level and harvest decisions for evenaged trees under uncertain future timber prices. They show that optimal thinning depends on stand age and other factors, and that there is an optimal time of final harvest.

When examining the literature, we find that there is a considerable amount of empirical field research done to show that thinning is very useful in order to improve the overall quality of specific crops and that it can optimize 
the economic returns pertaining to growers. This practical research is available for a variety of agricultural crops, fruits, and also in viticulture.

In horticulture, crop thinning is primarily used to raise overall fruit quality. Martín [1] found that thinning of peach crops may be necessary to obtain high yields, and that there is an optimal balance between yield and fruit size, which may depend on factors like the initial crop load, fruit size potential, time of thinning, date of harvest, and on the price of the crop. They also found that crop thinning costs may be considerable and will depend on the thinning technique used (hand, mechanical, or chemical). Meland [10] analysed thinning at first bloom and at $20 \mathrm{~mm}$ fruit size, comparing fruit growth and colour of Elstar apples in Norway and found that thinning at first bloom resulted in significantly higher fruit weights and improved background fruit colour. Lang and Ophardt [11] report that flower bud thinning in cherries prior to bloom although decreasing total yield up to $25 \%$ increased average fruit size up to $43 \%$.

In viticulture, field research has shown that there is a relatively strong link between cluster thinning and grape quality. For example, it has been shown that cluster thinning, its intensity and water (irrigation) conditions influences the quality of Tempranillo grapes $[12,13]$. In addition, it has been shown recently that cluster thinning also affects the subsequent quality of wine from Syrah grapes under Mediterranean climatic conditions [14].

Inserting economic considerations, research has shown that there is also a strong link between crop load, grape quality and resulting prices, which has significant implications for overall returns from a vineyard and attainable wine prices [15]. Ough and Nagaoka [4] found that the quality of California Cabernet Sauvignon was slightly higher when the crop was thinned in at least two out of three years. Sun et al. [5] found that wine growers implementing specific cluster thinning practices would have to receive higher bottle prices (between \$0.02-\$0.41) to compensate for additional labour costs and lost yield in order to maintain their economic welfare. In an applied technical brief, Preszler et al. [16] develop a static model allowing grape growers to quantify optimal yields and prices in order to enhance the decision-making accuracy and the economic sustainability of vineyard operations. Moreover, they apply this framework and show that cluster thinning may reduce the economic viability of Riesling production in New York State [17].

We note that a model of crop thinning seems to be highly relevant in viticulture as a lot of empirical studies have been applied in this field to evaluate the relationship between crop load and quality. However, to the best of our knowledge there is no theoretical model analysing the economic relationships between crop load, quality and optimal economic outcomes using a dynamic optimal control approach. We propose a first model to close this gap in the literature. While our approach is more general, we frame it in terms of viticulture and winemaking due to the prevalence of applied scientific studies in this field.

\section{Optimal control model}

To study how crop or cluster thinning can be done in an optimal way, we apply a dynamic model, based on optimal control theory. We would like to point out that our model differs from thinning models applied to forestry problems. Unlike in the case of timber, our model assumes that the planning horizon is fixed by nature (i.e. the problem of optimal rotation length does not emerge), there are no different aged crops in the field and the crop load which is thinned away is not marketable and has to be thrown away. For a more detailed discussion of the forestry literature on harvesting and rotation lengths, see Newman [18]. Moreover, for the sake of simplicity we will assume that there is no uncertainty about demand conditions at terminal time T. Our model is therefore deterministic.

The land owner's problem is to maximize the profit from selling the crop, grown on a given and fixed land area, facing a downward sloping demand curve for crop at the end of the growing period. In other words, the crop owner has some price setting (monopoly) power. Reasons for this monopoly power can be that the crop owner is able to influence the quality perception of his product making it a particular varietal expression of the crop, that can be distinguished from other producers (monopolistic competition). For example, such a market is certainly given for high quality wine grapes or regional origin denominated horticultural products. The demand for the crop, $x$, depends negatively on the price and positively on the crops' quality. $x$ measures the quantity of crop (i.e. the number of berries), not its size, weight or quality. It is exogenous and equal to the quantity that can be harvested under optimal growing conditions (i.e. without natural decay). The inverse demand function for the crop is

$$
p(x, q) ; \quad p_{x}<0, p_{q}>0 .
$$

Crop quality $q$ is a function of crop quantity, and we reasonably assume that after some amount of crop, the more crop is grown on the given area of land, the lower the crop's quality. Formally, we introduce the crop quality function

$$
q=f(x) \quad f^{\prime}(x) \geq 0, f^{\prime \prime}(x)<0 .
$$

The owner has the possibility to cut crop in an attempt to increase its quality. Denoting $s$ the rate at which crop is cut, and $\delta$ the rate of natural decay of crop, the stock (quantity) of crop, $\delta$, evolves according to

$$
\dot{x}=-(s+\delta) x .
$$

Cutting crop and maintaining the crop field causes costs. The cost function is given as

$$
C(x, s) ; \quad C_{x}>0, C_{s}>0 .
$$

At the end of the planning/crop growing period, achieved at time $T$, the crop is sold at the market. The crop owner's problem to maximize profits $\Pi$ by choosing the time path of the cutting rate $s$, i.e.

$$
\Pi=p(x, f(x)) x(T) e^{-r T}-\int_{0}^{T} C(x, s) e^{-r t} d t,
$$

where $r$ is the interest rate applied to discount future costs and revenues, subject to 


$$
\begin{gathered}
\dot{x}=-(s+\delta) x \\
x(t) \geq 0 \\
s(t) \geq 0 \\
x(0)=x_{0} .
\end{gathered}
$$

The inequalities (2b) and (2c) state that both the stock of crop on the field and the cutting rate cannot become negative. Equation (2d) is the initial condition, that is the amount of crop given at time 0 .

To be able to perform a qualitative analysis, we apply the following functional forms for the inverse demand function, the crop quality function and the cost function

$$
\begin{gathered}
p(x, f(x))=f(x)-b x ; b>0 \\
f(x)=k+m x-n x^{2} ; k>0, m>0, n>0 \\
C(x, s)=\frac{\alpha}{2} x^{2}+\frac{\beta}{2} x s^{2} ; \quad \alpha>0, \beta>0
\end{gathered}
$$

where $\alpha, \beta, b, k, m$ and $n$ are positive constants. The inverse demand function (3a) has an ordinate intercept (i.e. a prohibitively high price) which depends on grape quality. Grape quality (3b) is modelled as a concave quadratic function. Grape quality first increases with the amount of crop and eventually decreases when crop quantity is above a certain threshold

$$
\breve{x}=\frac{m}{2 n} \text {. }
$$

Finally, the cost function is quadratic in the amount of crop grown on the given area of land, and the cutting costs depend on crop quantity and increase quadratically with the cutting rate, $s$. Note that Martín et al. (2010) found a close relationship between cost and field capacity, which, in our model, is approximated with crop quantity, $x$.

Using Eqs. (3a) and (3b), we can calculate the crop owner's marginal revenue as

$$
\operatorname{MR}(x)=k+2(m-b) x-3 n x^{2} .
$$

Marginal revenue is a concave quadratic function of crop quantity. Using the functional forms (3), the Hamiltonian of the crop owner is

$$
H \equiv-\frac{\alpha}{2} x^{2}-\frac{\beta}{2} x s^{2}-\lambda(s+\delta) x+\gamma_{1} x+\gamma_{2} s
$$

where $\lambda$ is the shadow value of crop, and $\gamma_{1}, \gamma_{2}$ are the Lagrange multipliers attached to the non-negativity constraints on the stock of crop on the field and on the cutting rate, respectively. The first order conditions of the crop owner's problem are

$$
\begin{gathered}
-\beta x s-\lambda x+\gamma_{2}=0 \\
\dot{\lambda}-r \lambda=\alpha x+\frac{\beta}{2} s^{2}+\lambda(s+\delta)-\gamma_{1}
\end{gathered}
$$

together with the complementary slackness conditions

$$
x \geq 0, \gamma_{1} \geq 0, \quad \gamma_{1} x=0 ; \quad s \geq 0, \gamma_{2} \geq 0, \gamma_{2} s=0
$$

and the terminal condition

$$
\lambda(T)=\operatorname{MR}(x(T))=k+2(m-b) x(T)-3 n x(T)^{2} .
$$

Note that as long as the cutting rate $s$ and the stock of crop $x$ are positive, the multipliers $\gamma_{1}, \gamma_{2}$ are zero. Equation (5a) equates the marginal cutting cost to the marginal value of cutting $\gamma_{2}$. Equation (5b) is a dynamic no-arbitrage condition, requiring that along an optimal path the rate of return on crop has to be equal to the interest rate. This can be seen by rewriting ( $5 b)$ as

$$
\frac{\dot{\lambda}}{\lambda}-\frac{1}{\lambda}\left(a x+\frac{\beta}{2} s^{2}-\gamma_{1}\right)-(s+\delta)=r .
$$

The left hand side represents the rate of return on crop, where the first term is the "capital gain," the second term is a "dividend yield" and the third term a loss due to cutting and decay. Note that as long as the stock of crop is positive, $\gamma_{1}=0$.

\section{Optimal thinning}

Assume that the cutting rate $s$ is initially positive (otherwise the problem would degenerate). Hence, $\gamma_{2}=0$. Also, let the initial stock of crop be positive, such that $\gamma_{1}=0$. In this case, we can solve Eq. (5a) for the cutting rate

$$
s=-\frac{\lambda}{\beta}
$$

and insert this into $(5 \mathrm{~b})$, yielding the equation of motion for the shadow value.

$$
\dot{\lambda}=\alpha x+\lambda(s+\delta)-\frac{1}{2 \beta} \lambda^{2}
$$

Substituting the cutting rate (6) into the equation of motion for crop (2a) gives

$$
\dot{x}=\left(\frac{\lambda}{\beta}-\delta\right) x .
$$

Equations (7a) and (7b) form the dynamic system, given the assumption that both the stock of crop and the cutting rate are positive. System (7) can be shown to have three steady-states, the values of which are denoted by a tilde:

$$
\begin{gathered}
\tilde{x}=0, \tilde{\lambda}=0 \\
\tilde{x}=0, \tilde{\lambda}=2 \beta(r+\delta) \\
\tilde{x}=-\beta \delta(r+0.5 \delta) / \alpha, \tilde{\lambda}=\beta \delta
\end{gathered}
$$

none of which, of course, will be actually achieved, if one follows the optimal policy, because a zero terminal stock of crop would imply zero revenues, and a negative terminal stock of crop would violate the non-negativity condition on crop, Eq. (2b). 


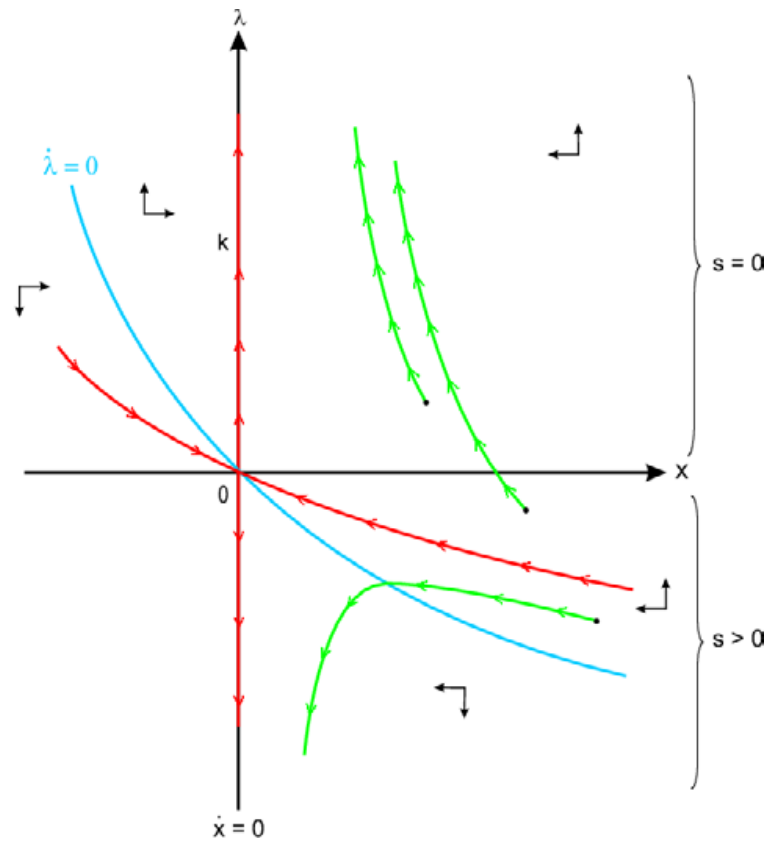

Figure 1. Phase Diagram with Paths.

In case that, say during transition, the cutting rate, s, becomes zero, while the stock of crop, $x$, remains positive (implying $\gamma_{1}=0$ ), Eqs. (5a) and (5b) become

$$
\begin{gathered}
\lambda x=\gamma_{2} \\
\dot{\lambda}=\alpha x+\lambda(r+\delta)
\end{gathered}
$$

and the equation of motion for the stock of crop is simply

$$
\dot{x}=-\delta x
$$

implying an exponential decay of crop. Then equation (9) simply determines the shadow value of cutting, $\gamma_{2}$. Equations (10a) and (10b) are a system of linear differential equations, the roots of which are $\mu_{1}=-\delta<0, \mu_{2}=r+\delta>0$. Hence the unique steadystate

$$
\tilde{x}=0, \tilde{\lambda}=0
$$

is saddle point stable. Taking the information provided by systems (7) and (10) together, we can construct a phase diagram for the relevant space, shown in Fig. 1. For the sake of clarity, we have not drawn in the other two steadystates $(8 \mathrm{~b})$ and $(8 \mathrm{c})$, because they are irrelevant.

The red arrowed curves are the stable and unstable branches of systems (7) and (10). We have drawn some trajectories. Any starting point located below the stable saddle going through the origin is unfeasible, as the shadow value of crop remains negative (note if there is some cutting $(s>0)$, the shadow value must be negative [see equation(6)] and the system's relevant steady state is given by equation (8a), which is the origin). Thus, a candidate for a starting point, given the initial stock of crop $x(0)=x_{0}$ has to be located above the stable branch (positions on the stable branch are not optimal, as it is not the goal to achieve the steady-state with a zero stock of crop). Hence, we have to determine the initial value of the jump variable $\lambda$, and thus we have to figure out which path the crop owner should follow, we have to implement the

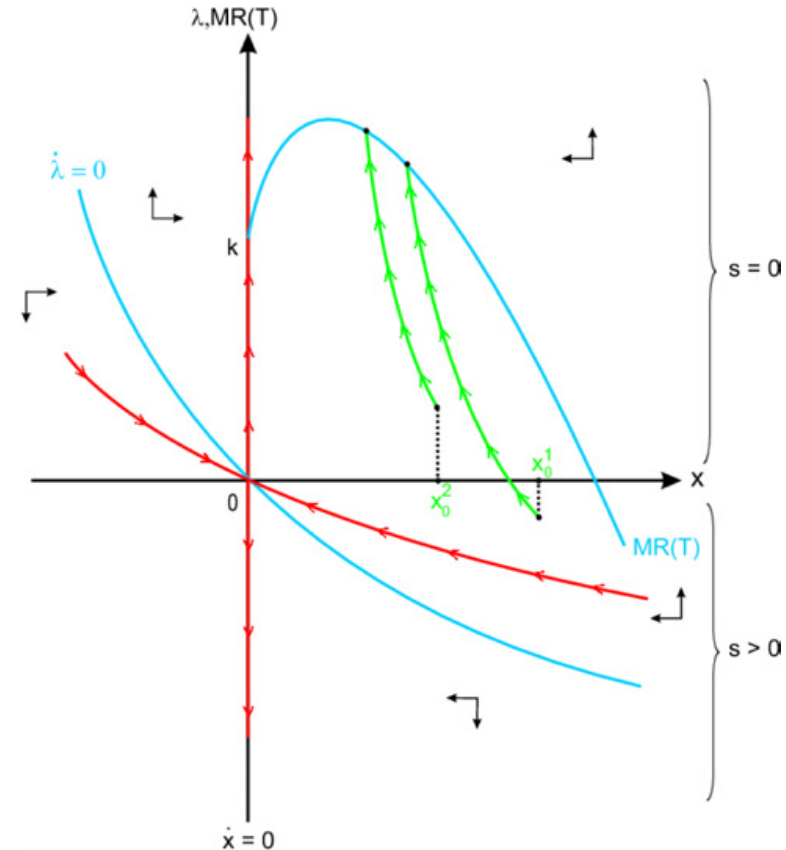

Figure 2. Phase Diagram with MR.

terminal condition $(5 \mathrm{c})$, requiring that the shadow value of crop is equal to its marginal revenue. Adding the marginal revenue curve to Fig. 1 results in Fig. 2.

The terminal condition $(5 \mathrm{c})$ requires that at terminal time $T$, the trajectory has to meet exactly the marginal revenue curve. From Fig. 2, we can identify two possible outcome scenarios:

a) If the intial quantity of the crop, e.g. $x(0)=x_{0}^{2}$, is sufficiently small, it is optimal not to thin it, but to simply let it evolve over time and to harvest and market it at time $T$. In this case, the shadow value of crop must be positive right at time zero, such that stocks of the crop simply evolve according to Eq. (10b) and decay exponentially at rate $\delta$. The correct initial shadow value $\lambda(0)$ is determined by the condition such that the trajectory meets the marginal revenue curve at terminal time $T$. Not thinning in turn keeps the crop owner's costs as low as possible, thus contributing to his intertemporal profit.

b) If the initial stock of crop is high, e.g. $x(0)=x_{0}^{1}$, then the owner's optimal policy has two stages. In the first stage, at the beginning of the planning horizon, it is optimal to cut crop, however at a decreasing rate, as the trajectory confirms. Together with natural decay, the stock of crop shrinks over time and its shadow value becomes less negative. At some point in time, the trajectory crosses the horizontal axis, and the shadow value of crop becomes zero. At that point, the second stage begins. It is now optimal to stop cutting and let the crop simply shrink according to nature. The shadow value of crop becomes positive and increases over time. Eventually, at terminal time $T$, the trajectory reaches the marginal revenue curve. The crop is then harvested and sold on the market.

There is a third, but less interesting possibility. It may turn out that both scenarios above, even though they maximize the crop owner's intertemporal profit, result in an overall 
loss. In this case, vineyard owners would simply shut down their operations and leave the land idle. In this case, intertemporal profits would be zero.

It is important to note that the case actually prevailing depends on the model's parameters. For example, if the quality function does not sufficiently strongly depend on quantity $\mathrm{x}$, (in other words, the consumers' willingness to pay is not strongly affected by quality), it may turn out that cluster thinning is never optimal.

\section{Conclusions}

Developing a simple optimal control model for the crop owner's profit maximization problem, we found three possible outcomes for the decision whether a crop should be thinned or not. First, if the initial crop quantity is sufficiently small, then it is optimal not to thin the crop, but simply to let it evolve over time and to harvest and market them at time T. Second, if initial crop quantity is high, then it is optimal to cut crop at the beginning of the planning horizon and to reduce cutting (i.e. the cutting rate $s$ falls) over time until the stock of crop has arrived at its optimum. Depending on the model's parameters, this "stopping time" is reached sooner or later. From thereon, quantity evolves solely according to natural decay. Third, and less relevant, if intertemporal profits are negative, it is optimal to shut down the production.

In this paper, we have developed a general optimal control model of crop or cluster thinning. It fits observed behavior in viticulture but also for other horticultural crops (e.g. apples, peaches, cherries). Our results may be linked to a number of papers in the science literature, which underscores the applicability of our dynamic model. For example, both Preszler et al. [17] as well as our outcome scenario b) indicate that thinning may not be profitable. Moreover, Chapman et al. [19] show that quality (i.e. aromas and flavours) respond significantly to thinning, but "only when yield is altered early in fruit development," a result that can be linked to our outcome scenario b) which indicates that thinning is to be done at the beginning of the planning horizon. In addition, incremental changes as in our dynamic model directly relate to the Gil et al. study [20], indicating that small changes in crop size due to individual berry thinning instead of whole cluster thinning may yield significant and more pronounced improvements in wine quality and profitability.

A limitation of the theoretical model is that it is in continuous time rather than in discrete time. However, its general conclusion to reduce thinning over time to arrive at an optimal stock of the crop would remain valid. Another limitation of this model is that it is deterministic in nature. Introducing uncertainty would be an interesting extension to the model. Moreover, as the model is simplified and demand for the crop $x$ is given and only varies due to thinning and natural decay, it seems worthwhile to generalize this in the future. Finally, the model implicitly imposes the requirement that all of the existing crop at terminal time $\mathrm{T}$ has to be sold on the market. A possible extension would be to allow for the possibility to sell only part of the crop and to discard the remaining crop, aiming at "riding the demand curve." Moreover, future research employing dynamic optimal control modelling should also empirically validate our results beyond the links to previous scientific research in viticulture that we established in this paper.

\section{References}

[1] B. Martín, A. Torregrosa, J. Garcia Brunton (2010): Post-bloom thinning of peaches for canning with hand-held mechanical devices, Sci. Hortic. 125, 4 658-665

[2] L.A. Jozsa, H. Brix (1989): The effects of fertilization and thinning on wood quality of a 24-year-old Douglas-fir stand. Can. J. For. Res. 19, 9: $1137-1145$

[3] R.A. Fischer, D.R. Laing (1976): Yield potential in a dwarf spring wheat and response to crop thinning. J. Agr. Sci. 87: 113-122

[4] C.S. Ough, R. Nagaoka (1984): Effect of Cluster Thinning and Vineyard Yields on Grape and Wine Composition and Wine Quality of Cabernet Sauvignon. Am. J. Enol. Vitic. 35, 1: 30-34

[5] Q. Sun, G.L. Sacks, S.D. Lerch, J.E. Vanden Heuvel (2012): Impact of Shoot and Cluster Thinning on Yield, Fruit Composition, and Wine Quality of Corot noir. Am. J. Enol. Vitic. 63, 1: 49-56

[6] G.F. Schreuder (1971): The simultaneous determination of optimal thinning schedule and rotation for an even-aged forest, For. Sci. 17, 3: 333-339

[7] J.L. Medhurst, E.A. Pinkard, C.L. Beadle, D. Worledge (2003): Growth and stem form responses of plantation-grown Acacia melanoxylon (R.Br.) to form pruning and nurse-crop thinning, For. Ecol. Manage. 179, 1: 183-193

[8] M. Sourcy (2011): Influence of wood value on optimal type of thinning, Paper prepared for the 34th Council on Forest Engineering, Quebec City, Canada

[9] F. Lu, P. Gong (2003): Optimal stocking level and final harvest age with stochastic prices, J. For. Econ. 9, 2: 119-136

[10] M. Meland (2009): Effects of different crop loads and thinning times on yield, fruit quality, and return bloom in Malus x domestica Borkh. 'Elstar'. J. Hortic. Sci. Biotechnol. ISAFRUIT Special Issue: 117-121

[11] G.A. Lang, D.R. Ophardt (2000): Intensive crop regulation strategies in sweet cherries. Acta Horticulturae 514, Proc XXV IHC - Part 4

[12] L.G. Santesteban, C. Miranda, J.B. Royo (2011): Thinning intensity and water regime affect the impact cluster thinning has on grape quality. Vitis: 50, 4: 159-165

[13] E. Gamero, D. Moreno, I. Talaverano, M.H. Prieto, M.T. Guerra, M.E. Valdés (2014): Effects of irrigation and cluster thinning on Tempranillo grape and wine composition. S. Afr. J. Enol. Vitic. 35, 2: 196-204

[14] C. Condurso, F. Cincotta, G. Tripodi, A. Sparacio, D.M.L. Giglio, S. Sparla, A. Verzera (2016): Effects of cluster thinning on wine quality of Syrah cultivar (Vitis vinifera L.), Eur. Food Res. Technol. 242, 10: $1719-1726$

[15] A. Calderon-Orellana, M.A. Matthews, W.M. Drayton, K.A. Shackel (2014): Uniformity of ripeness and size in Cabernet Sauvignon berries 
from vineyards with contrasting crop price, Am. J. Enol. Vitic. 65, 1: 81-88

[16] T. Preszler, T.M. Schmit, J.E. Vanden Heuvel (2010): A model to establish economically sustainable cluster thinning practices. Am. J. Enol. Vitic. 61, 1: 140-146

[17] T. Preszler, T.M. Schmit, J.E. Vanden Heuvel (2013): Cluster thinning reduces the economic sustainability of Riesling production. Am. J. Enol. Vitic. 64, 3: 333-341

[18] D.H. Newman (2002): Forestry's golden rule and the development on the optimal forest rotation literature, J. For. Econ. 8: 5-27
[19] D.M. Chapman, M.A. Matthews, J.-X. Guinard (2004): Sensory Attributes of Cabernet Sauvignon Wines Made from Vines with Different Crop Yields. Am. J. Enol. Vitic. 55, 4: 325-334

[20] M. Gil, M. Esteruelas, E. González, N. Kontoudakis, J. Jiménez, F. Fort, J.M. Canals, I. Hermosín-Gutiérrez, F. Zamora (2013): Effect of two different treatments for reducing grape yield in vitis vinifera cv Syrah on wine composition and quality: Berry Thinning versus Cluster Thinning, J. Agric. Food Chem, 61, 20: 4968-4978 